\title{
ARTICLE
}

\section{Comparison of the Monte Carlo Adjoint-Weighted and Differential Operator Perturbation Methods}

\author{
Brian C. KIEDROWSKI ${ }^{*}$ and Forrest B. BROWN \\ Los Alamos National Laboratory, Los Alamos, NM, 87545, USA
}

\begin{abstract}
Two perturbation theory methodologies are implemented for $k$-eigenvalue calculations in the continuous-energy Monte Carlo code, MCNP6. A comparison of the accuracy of these techniques, the differential operator and adjoint-weighted methods, is performed numerically and analytically. The results show that neither method is, in general, more accurate.
\end{abstract}

KEYWORDS: MCNP, criticality, perturbation theory, adjoint flux, differential operator, Taylor series

\section{Introduction}

Methods of perturbation theory allow for the fast calculation of changes in a response from small changes to a system. In the early days of computing, these techniques were invaluable since exploring the parameter space using direct calculations was prohibitive. Today, computers allow Monte Carlo methods to be a more practical design tool; nonetheless, such calculations are often expensive and a cheap way to explore a design space, albeit approximately, is desirable.

MCNP5 $^{1)}$ computes changes in a tally response (such as $k$-eigenvalue) from a prescribed perturbation using the differential operator technique. ${ }^{2)}$ Version 6 of MCNP will feature the adjoint-weighted methodology ${ }^{3-4)}$ for calculating changes in reactivity strictly in $k$-eigenvalue problems.

With these two methods available, a discussion of which is most accurate is appropriate. To determine this, perturbations in $k$ (the $k$-eigenvalue) are estimated using both techniques and compared against reference solutions generated from a direct calculation by subtracting results of $k$ for from the perturbed and unperturbed cases. Next, analytic solutions to a simple problem are generated that demonstrate successes and failures of both methods.

\section{The Differential Operator Technique}

The premise of the differential operator technique is centered on a Taylor series expansion. It is assumed that the nuclear cross section $\sigma$ has the form of $\exp (\theta)$ where $\theta$ is some dimensionless parameter. The change in $k$ from a perturbation $\Delta \theta$, is:

$$
\Delta k=\frac{\partial k}{\partial \theta} \Delta \theta+\frac{1}{2} \frac{\partial^{2} k}{\partial \theta^{2}}(\Delta \theta)^{2}+\ldots
$$

The change in the parameter $\Delta \theta$ is the change in the relative cross section. In MCNP, Eq. (1) is truncated with either one or two terms (first- or second-order perturbation theory).

*Corresponding author, E-mail:bckiedro@lanl.gov

(C) 2011 Atomic Energy Society of Japan, All Rights Reserved.
There are Talyor terms for each perturbed nuclide. Note that no cross-terms between derivatives of nuclides are considered. This has been shown to have an impact upon the accuracy of results in some problems. ${ }^{5)}$

To calculate the derivatives in Eq. (1), two terms must be estimated. The first is the derivative of the probability of the random walk occurring. The second is the derivative of the tally response itself. For $k$, the tally response for an individual track is $v \Sigma_{f} w L$. The nomenclature is as follows: $v$ is the average number of neutrons produced in a fission event, $\Sigma_{f}$ is the macroscopic fission cross section, $w$ is the particle simulation weight, and $L$ is the length of the track. The sum of all histories is normalized by the volume of the cell to produce a neutron production rate.

There is an additional derivative for changes in the fission source shape that MCNP does not account for. Previous work has shown that this may significantly impact the accuracy of results. ${ }^{6)}$

\section{The Adjoint-Weighted Technique}

Starting from the neutron transport equation and applying a first-order perturbation, the following expression for the change in reactivity $\rho$ can be derived: ${ }^{3)}$

$$
\Delta \rho=-\frac{\left\langle\psi^{\dagger}, P \psi\right\rangle}{\left\langle\psi^{\dagger}, F^{\prime} \psi\right\rangle}
$$

The reactivity is related to $k$ in the typical way, $\rho=$ $(k-1) / k$. The angular flux in the unperturbed system is $\psi$ and its adjoint is denoted by $\psi^{\dagger} . P$ is the operator for the perturbation taking the form: $P=\Delta \Sigma_{t}-\Delta S-\lambda \Delta F$. The eigenvalue $\lambda=1 / k$, and the three terms from left to right, are the change in the total cross section, the change in the scattering operator, and the change in the fission multiplication operator. $F^{\prime}$ is the perturbed fission operator.

Monte Carlo techniques can be used to sample the numerator and the denominator in a continuous-energy forward calculation $^{4)}$ and the change in reactivity can be estimated by taking the ratio in Eq. (2). Note that the implementation in 


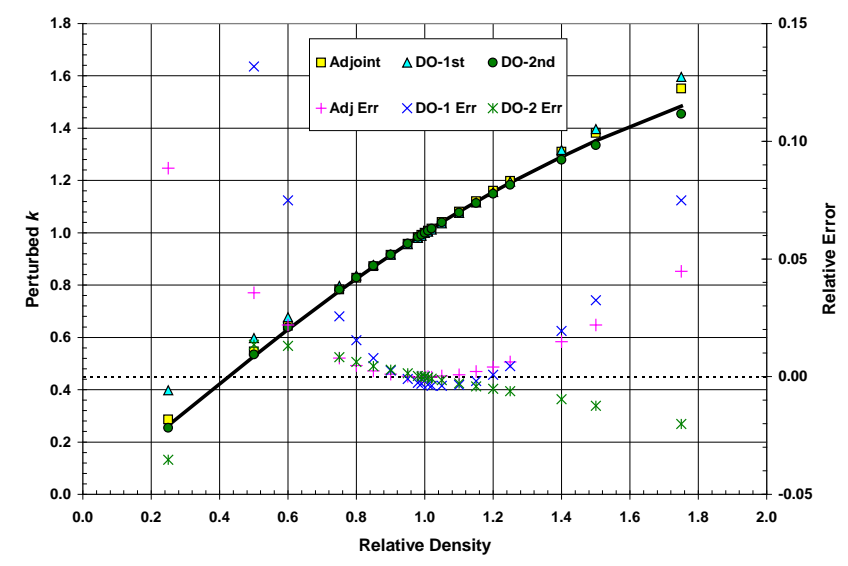

Fig. 1 Results of perturbation theory methods and relative errors compared to reference solutions for the global density perturbation in Godiva

MCNP does not take into account the perturbation in scattering laws. In applications involving calculating perturbations for specific reactions of specific isotopes (such as in generating sensitivity coefficients), there is evidence to suggest this approximation introduces significant sources of error $^{7}$.

\section{Numerical Results}

Continuous-energy, $k$-eigenvalue problems are run using MCNP6. Both the differential operator and adjoint-weighted perturbation methods (eight latent generations, which is found to be adequate from sensitivity studies ${ }^{4}$ ) are employed to estimate the change in $k$. A reference value is obtained by subtracting the results of two independent MCNP6 calculations.

Two problems of interest are presented. The first is Godiva ${ }^{8)}$, a bare sphere of high-enriched uranium (HEU) with radius $8.74 \mathrm{~cm}$. The other is a 2-D, quarter-core pressurized water reactor (PWR) model. ${ }^{9}$ ) Both problems use ENDF/B-VII.0 nuclear data. In all cases, statistical uncertainties are less than one percent.

\section{Godiva}

\section{(1) Global Density}

The tests with Godiva involve changes in density (globally as well as locally) and changes in enrichment. For the first test, the density of the HEU metal is varied from $25 \%$ to $175 \%$ of the nominal density. Perturbed results of $k$ are obtained for various density perturbations for both methods (the differential operator has curves for both first- and second-order perturbations); these and the reference $k$ from a direct calculation are compared in Fig. 1.

To better compare the results, Fig. 1 gives, on the right-axis, the relative error (not to be confused with the Monte Carlo uncertainties) with respect to the reference $k$.

The most accurate method for this perturbation is the second-order differential operator method, followed by the adjoint-weighted approach. This is true over the entire domain of the density perturbation.

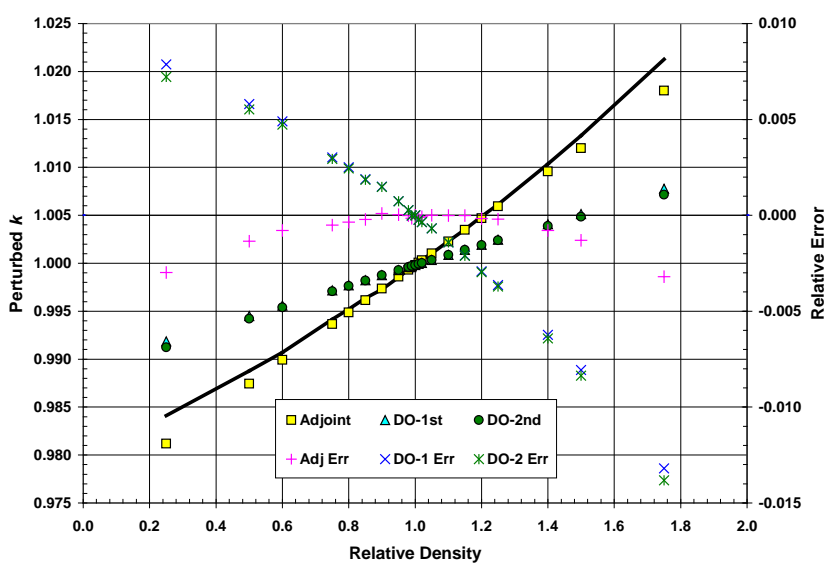

Fig. 2 Results of perturbation theory methods and relative errors compared to reference solutions for the localized density perturbation (inner $2 \mathrm{~cm}$ ) in Godiva

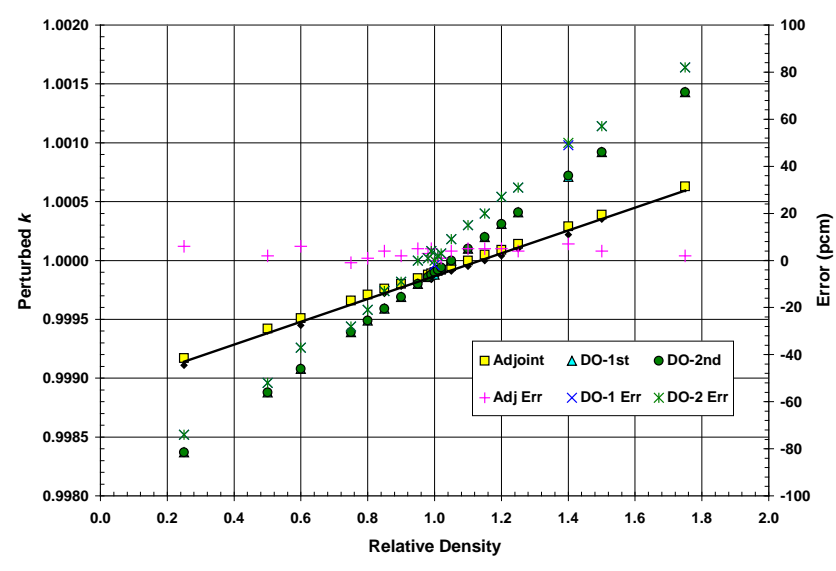

Fig. 3 Results of perturbation theory methods and absolute errors compared to reference solutions for the localized density perturbation (outer $0.01 \mathrm{~cm}$ ) in Godiva

\section{(2) Localized Inner Density}

The same experiment is carried out, but this time only the density of the central $2 \mathrm{~cm}$ of Godiva is perturbed. Figure 2 displays the relative errors for each of the methods. Both methods are able to capture the perturbed $k$ within one percent with the adjoint-weighted approach being more accurate over a wider range.

Interesting is the difference in shape between the curves produced by the different methods. In this case, the adjoint-weighted method always predicts a perturbed $k$ that is too low, whereas the differential operator method predicts $k$ being too high for reductions in density and too low for increases in density. While the sign of the error may be reversed, this trend in the error of predicting $k$ is observed for a wide variety of calculations.

(3) Localized Edge Density

Like with the previous, the density is perturbed, but this time only in the outer $0.01 \mathrm{~cm}$ of the sphere. ${ }^{10)}$ The results of $k$ along with the errors (this time absolute) in pcm $(1 \mathrm{pcm}=$ $1 \times 10^{-5}$ ) are displayed in Fig. 3. Because the effect of this perturbation is relatively small, even statistical uncertainties of 1 to $2 \mathrm{pcm}$ can distort the curve produced. For this reason, a linear fit is applied with an R-squared value of 0.9951 . 


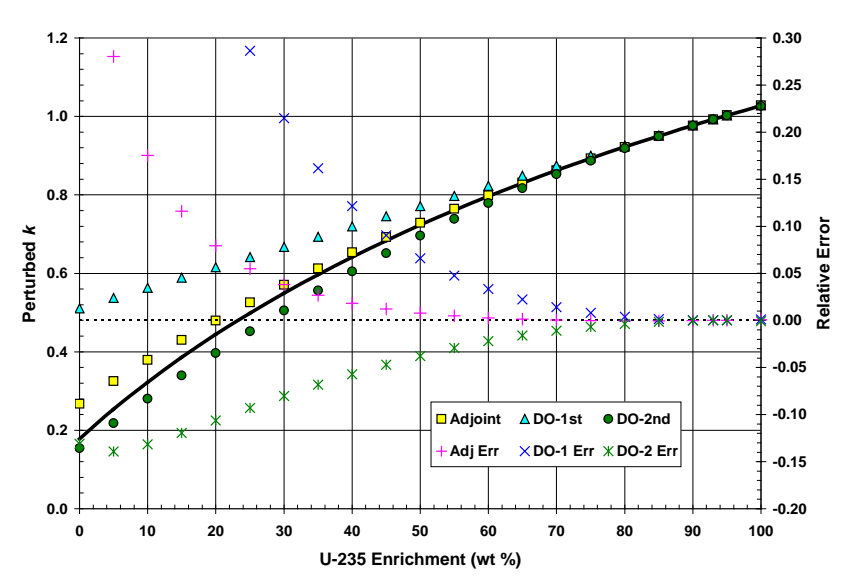

Fig. 4 Results of perturbation theory methods and relative errors compared to reference solutions for the ${ }^{235} \mathrm{U}$ enrichment perturbation in Godiva

This appears justified as both the first- and second-order differential operator method are nearly identical, implying the perturbation can be described with only the first, linear Taylor term.

The adjoint-weighted method follows the true $k$ closely (there is noise because of statistical uncertainties) whereas both first- and second-order differential operator values of $k$ follow a line with an incorrect slope.

It has been shown ${ }^{6)}$ that this is because MCNP does not currently handle the perturbation in the fission source and that doing so largely addresses this problem. The adjoint-weighted approach, on the other hand, automatically accounts for the perturbation in the fission source. ${ }^{11)} \mathrm{A}$ fair comparison would require the implementation of the correction.

\section{(4) Enrichment}

A simplified model of the Godiva sphere is constructed with 93 wt $\%{ }^{235} \mathrm{U}$ and $7 \mathrm{wt} \%{ }^{238} \mathrm{U}$. The perturbation varies the enrichments from $0 \%$ to $100 \%{ }^{235} \mathrm{U}$ in $5 \%$ increments.

The perturbed values of $k$ and the corresponding relative errors (those in excess of $30 \%$ are not shown to better view performance for smaller perturbations) for the perturbation methods are displayed in Fig. 4. All methods appear to be quite accurate for enrichments around $80 \%$. For decreasing enrichment down to about $25 \%$, the adjoint-weighted method is the most accurate. Below that, the second-order differential operator appears to be best.

Generally speaking, typical perturbations would be on the order of a few tens of percent at most, and adjoint-weighted perturbation theory appears to function best in that regime. Note that the differential operator results are more accurate if the second-order cross-terms are approximated, ${ }^{5)}$ which they are not in MCNP5.

\section{Pressurized Water Reactor}

\section{(1) Boron Concentration}

The boron-10 concentration in the water region of the 2-D PWR model is varied. The reference concentration of ${ }^{10} \mathrm{~B}$ is $16.75 \mathrm{ppm}$, making the reactor approximately critical. Results of perturbed $k$ and relative errors (those in excess of

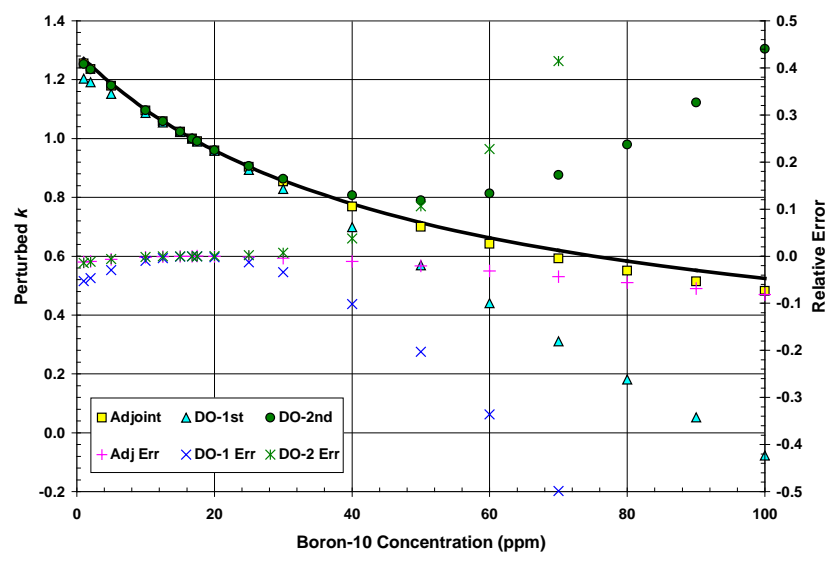

Fig. 5 Results of perturbation theory methods and relative errors compared to reference solutions for perturbing the boron-10 concentration in the coolant/moderator of a PWR

50\% are not displayed) are given in Fig. 5 for different concentrations from 1 to $100 \mathrm{ppm}$.

Only the adjoint-weighted perturbation theory is able to capture the boron-10 concentration perturbations within 10\% accuracy. Both the linear and quadratic curves formed by first- and second-order differential operator exceed $10 \%$ error for perturbations in excess of 40 and $50 \mathrm{ppm}$ of boron-10 respectively. Furthermore, both differential operator approximations produce unphysical behavior (in the case of first order, $k$ becomes negative, and, in the case of second order, $k$ begins to increase with adding boron-10). For differential operator to be more accurate, higher-order terms will be required.

(2) Xenon Concentration

Xenon-135 is distributed uniformly throughout the fuel. The reference concentration of ${ }^{135} \mathrm{Xe}$ is taken to be at $10 \mathrm{ppb}$. The perturbations vary the concentration from no xenon to $50 \mathrm{ppb}$. Perturbed $k$ results along with relative errors are displayed in Fig. 6. All methods appear to be able to capture the perturbation within three percent, with the second-order differential operator being the most accurate.

An important point is that had the unperturbed case been a fresh core (no xenon-135 present), the differential operator method would have been unable to compute any perturbations in ${ }^{135} \mathrm{Xe}$. This is because of the assumption that the nuclear cross sections vary as the exponential of some dimensionless parameter $\theta$. Therefore, differential operator cannot add a new impurity; the adjoint-weighted method does not have this restriction.

(3) Moderator Density

The next test is to see how well the various methods can predict $k$ for adding and removing moderation to the core. The as is, simplified model is strongly overmoderated with the current boron-10 concentration in the water. Because of this, changing the moderator density would more repeat the previous boron-10 concentration perturbation rather than look at the effect of adding/removing moderator.

The model is modified in two ways to produce a perturbation that tries to measure the change of core moderation. First, all boron-10 is removed from the water. Secondly, 


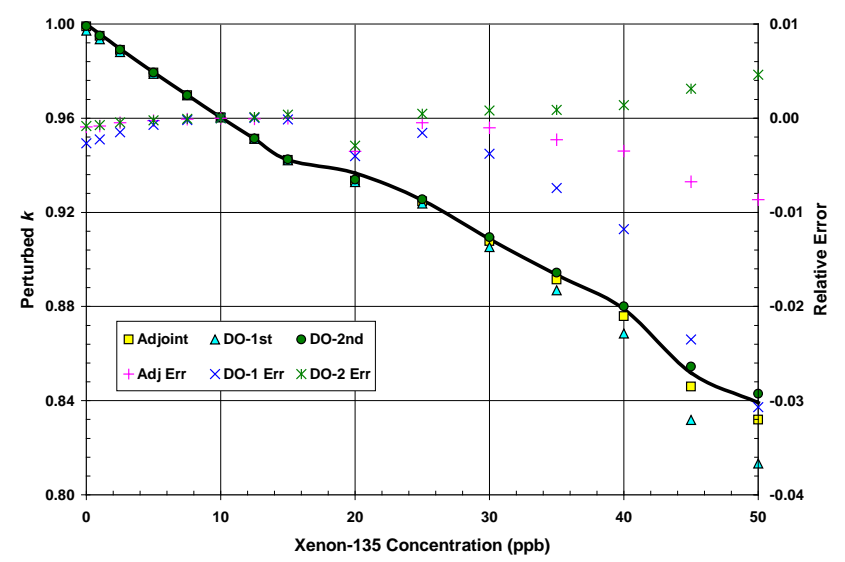

Fig. 6 Results of perturbation theory methods and relative errors compared to reference solutions for perturbing the xenon-135 concentration uniformly in the fuel of a PWR

$50 \mathrm{ppb}$ of xenon-135 is added uniformly throughout the fuel to bring $k$ back to near critical $(k \approx 1.024)$.

The density of the moderator is varied globally by $+/-25 \%$. The perturbations of $k$ are displayed in Fig. 7 along with the relative errors. In this case, the second-order differential operator is more accurate over this domain. Both the first-order differential operator and the adjoint-weighted perturbation theory are about the same in terms of accuracy.

\section{Analytical Comparisons}

The change in $k$ is calculated exactly for changes in the capture and scattering cross sections of a simple two-group, infinite medium problem (unperturbed $k=1$ ). The cross section data is given in Table 1. The analytic solution for $k$ is

$$
k=\frac{v \Sigma_{f 1}}{\Sigma_{R 1}}+\frac{v \Sigma_{f 2}}{\Sigma_{R 2}} \frac{\Sigma_{s 12}}{\Sigma_{R 1}}=1 .
$$

The removal cross section for energy group $g$ is defined as $\Sigma_{R g}=\Sigma_{t g}-\Sigma_{s g g}$, where $\Sigma_{t g}$ and $\Sigma_{s g g}$ are the total and within-group macroscopic scattering cross sections respectively for group $g$. $v \Sigma_{f g}$ is the mean neutrons per fission times the macroscopic fission cross section for group $g$ and $\Sigma_{s 12}$ is the group 1-to-2 scattering macroscopic cross section.

Exact, reference solutions for $k$ for various perturbations can be computed by directly evaluating Eq. (3) with modified cross section data.

To evaluate the analytic result for the differential operator technique, the expression in Eq. (3) is differentiated with respect to various cross sections. Numerical values for first and second derivatives of each cross section are given in Table 2. These derivatives are inserted into a Taylor expansion with respect to Eq. (3) and evaluated for a range of perturbations ranging from $+/-100 \%$ of the unperturbed cross section.

To compare adjoint-weighted perturbation results, Eq. (2) must be evaluated. This solves for $\rho$, which can easily be converted to $k$. To solve Eq. (2), the forward and adjoint fluxes must be computed from the transport equation. Since the system is an eigenvalue problem, there is a free parame-

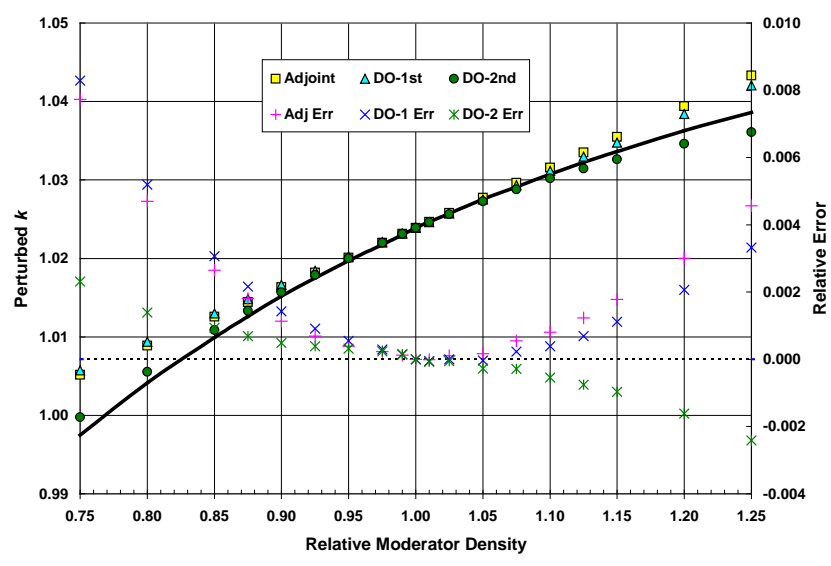

Fig. 7 Results of perturbation theory methods and relative errors compared to reference solutions for perturbing the moderator density throughout the PWR

Table 1 Cross-section data $\left(\mathrm{cm}^{-1}\right)$ for the two-group infinite-medium problem

\begin{tabular}{cccccccc}
\hline $\mathrm{g}$ & $\Sigma_{t}$ & $\Sigma_{c}$ & $\Sigma_{f}$ & $v$ & $\chi$ & $\Sigma_{s g 1}$ & $\Sigma_{s g 2}$ \\
\hline 1 & 2 & $1 / 2$ & $1 / 2$ & $3 / 4$ & 1 & $1 / 2$ & $1 / 2$ \\
\hline 2 & 3 & 1 & 1 & $9 / 2$ & 0 & 0 & 1 \\
\hline
\end{tabular}

Table 2 Numerical evaluations of derivatives of Eq. (3) with respect to cross sections

\begin{tabular}{ccc}
\hline & $\begin{array}{c}\text { First } \\
\text { Deriv. }\end{array}$ & $\begin{array}{c}\text { Second } \\
\text { Deriv. }\end{array}$ \\
\hline$\Sigma_{c 1}$ & $-2 / 3$ & $8 / 9$ \\
\hline$\Sigma_{c 2}$ & $-3 / 8$ & $3 / 16$ \\
\hline$\Sigma_{f 1}$ & $-1 / 6$ & $2 / 9$ \\
\hline$\Sigma_{f 2}$ & $3 / 8$ & $-3 / 8$ \\
\hline$\Sigma_{s 12}$ & $5 / 6$ & $-10 / 9$ \\
\hline
\end{tabular}

ter to normalize the equations; choose $\psi_{1}=1$ and $\psi_{2}^{\dagger}=1$.

From these definitions, the forward and adjoint fluxes for the other group can be determined to be

$$
\begin{aligned}
& \psi_{2}=\frac{\Sigma_{s 12}}{\Sigma_{R 2}}, \\
& \psi_{1}^{\dagger}=\frac{\Sigma_{R 2}}{v \Sigma_{f 2}} .
\end{aligned}
$$

Both the Taylor expansion in Eq. (1) and the relationship in Eq. (2) are used to calculate $\Delta k$ for perturbations of various magnitudes for the capture, fission, and group-to-group scattering cross sections. These computed values are then compared to reference solutions obtained from Eq. (3).

The relative errors of the perturbations for the capture cross sections are shown in Fig. 8. For the group-1 capture cross section, adjoint-weighted perturbation theory calculates the perturbed $k$ exactly. In the case of group-2, the adjoint-weighted perturbation is no longer exact, but is still more accurate than either the first- or second-order differential operator predictions. 


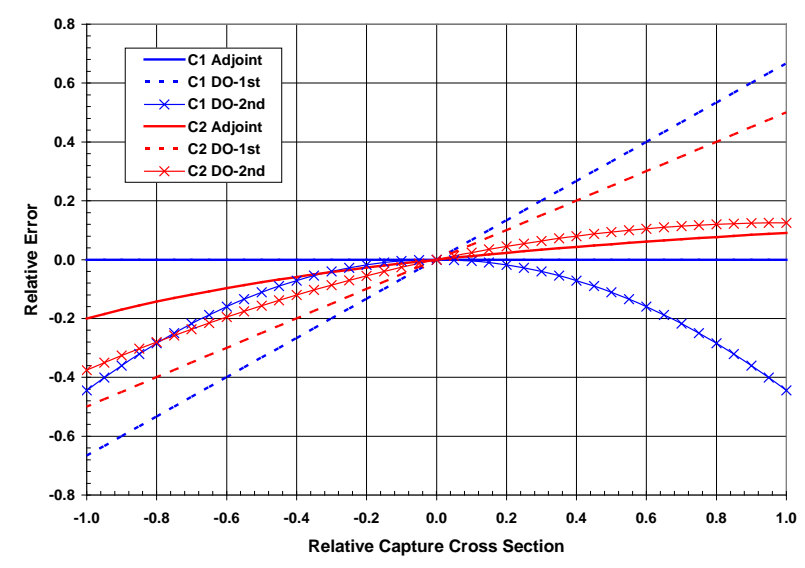

Fig. 8 Analytically derived relative errors for perturbing the capture cross sections in energy groups 1 and 2

The relative errors for $\Delta k$ of the fission cross section perturbations from the two methods are displayed in Fig. 9. Like with the capture cross section of group 1, the adjoint-weighted perturbation for the group 1 fission cross section is exact. The second-order differential operator method is more accurate for perturbations where the group 2 fission cross section is reduced from $0-60 \%$. However, the adjoint-weighted method still appears to be most accurate over a greater range.

Figure 10 shows the relative errors of $\Delta k$ for the group 1-to-2 scattering cross section perturbation. This case has both the first- and second-order differential operator method being superior to the adjoint-weighted method, opposite of the behavior observed in both the capture and fission cross section perturbations.

This perturbation is particularly pathological for the adjoint-weighted method. The change in reactivity predicted from adjoint-weighted perturbation theory is

$$
\Delta \rho=\frac{\left(1-\frac{\Sigma_{R 2}}{v \Sigma_{f 2}}\right) \Delta \Sigma_{s 12}}{\frac{v \Sigma_{f 1}}{v \Sigma_{f 2}} \Sigma_{R 2}+\Sigma_{s 12}} .
$$

When $\Sigma_{R 2} / v \Sigma_{f 2}<1, \rho$ can increase without bound for an arbitrarily large increase in the group 1-to-2 scattering cross section. The physical range of the reactivity is $-\infty<\rho<1$, where the lower and upper bounds correspond to a zero and infinite $k$ respectively. Should $\rho$ exceed unity, as is possible in Eq. (6), the results become unphysical. Note that is not a feature or weakness of MCNP or a Monte Carlo implementation, but of the theory itself. Applying adjoint-weighted perturbation theory to one such as this may produce vastly incorrect and even nonsensical results.

\section{Conclusions \& Recommendations}

Perturbation results from MCNP6 are compared against direct Monte Carlo calculations and analytic solutions. The results of these calculations show that, a priori, neither the differential operator method nor the adjoint-weighted ap-

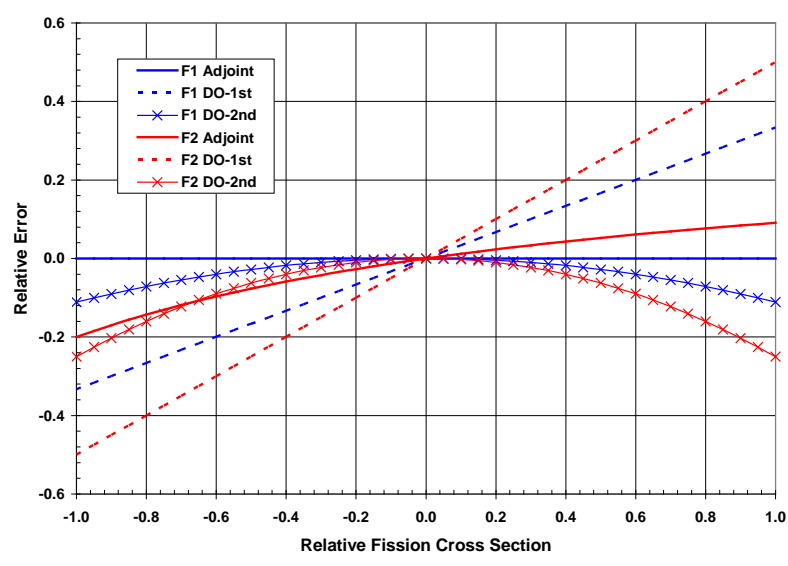

Fig. 9 Analytically derived relative errors for perturbing the fission cross sections in energy groups 1 and 2

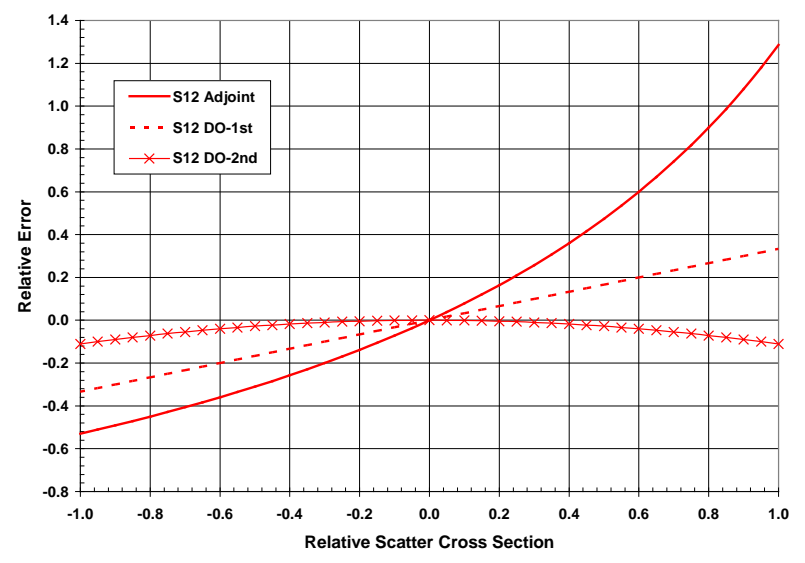

Fig. 10 Analytically derived relative errors for perturbing the group 1-to-2 scattering cross section

proach is better in all cases.

There is evidence to suggest adjoint-weighted perturbation theory will yield more accurate results most of the time. For some simple cases, adjoint-weighted perturbation theory produces exact results. However, there are notable exceptions and even pathological cases (e.g. the group 1-to-2 scattering perturbation in the infinite medium problem) where adjoint-weighted perturbation theory will always produce results far less accurate than even the first-order differential operator approach.

Users are therefore urged to exercise caution when using either perturbation theory method. This should come as no surprise, as perturbation theory is, by definition, inherently approximate except in simple cases. Never should one assert that either method, in general, produces results more accurate than the other. Nonetheless, perturbation theory provides a useful tool to efficiently probe a design space or compute sensitivity coefficients and its use is encouraged to this end so long as care is taken.

A future subject of study is determining conditions for which each method is appropriate, or, conversely, types of calculations that are best avoided with either method. Such diagnostics would be useful for informing a designer which method is most appropriate for the problem of interest. 
For MCNP6 (and other related codes) development, the following recommendations are made based on this research:

- The differential operator approach should account for perturbations to the fission source. This has been shown to greatly improve the accuracy of the calculation, albeit at added cost. $^{12)}$

- Higher-order and cross-terms for the differential operator should be developed and implemented; while this comes with increased cost, for some problems the cost may be justified. $^{12)}$

- For adjoint-weighted perturbation theory, the effect of changes to the scattering laws and fission emission spectra should be incorporated. ${ }^{7 \text { ) }}$

\section{Acknowledgments}

This research was performed by funding from the US Department of Energy Nuclear Criticality Safety program. Special thanks are given to Jeff Favorite at LANL for his insights.

\section{References}

1) X-5 Monte Carlo Team, MCNP - A general Monte Carlo $N$-Particle transport code, Volume I: Overview and theory, LA-UR-03-1987, Los Alamos National Laboratory (LANL) (2003).

2) H. Rief, "Generalized Monte Carlo perturbation algorithms for correlated sampling and second order Taylor series approach," Ann. Nuc. Enrgy., 11, 455 (1984).

3) E. E. Lewis, W. F. Miller, Jr., Computational Methods of Neutron Transport, American Nuclear Society, Inc., LaGrange Park, Illinois, USA, 52-55 (1993).

4) B. C. Kiedrowski, F. B. Brown, P. P .H. Wilson, “Calculating kinetics parameters and reactivity changes with conti- nuous-energy Monte Carlo,” Proc. PHYSOR 2010, Pittsburgh, Pennsylvania, USA, May 9-14, 2010 (2010), [CD-ROM].

5) J. A. Favorite, D. K. Parsons, "Second-order cross terms in Monte Carlo differential operator perturbation estimates,” Proc. International Conference on Mathematical Methods for Nuclear Applications, Salt Lake City, Utah, USA, Sept. 9-13, 2001 (2001), [CD-ROM].

6) Y. Nagaya, F. B. Brown, "Estimation of the change in k-effective due to perturbed fission source distribution in MCNP,” Proc. M\&C 2003, ANS Mathematics \& Computation Topical Meeting, Gatlinburg, Tennessee, USA, Apr. 6-10, 2003 (2003), [CD-ROM].

7) B. C. Kiedrowski, J. A. Favorite, F. B. Brown, "Verification of k-eigenvalue sensitivity coefficient calculations using adjoint-weighted perturbation theory in MCNP," Trans. Am. Soc., 103, 409-411 (2010).

8) J. B. Briggs (ed.), International Handbook of Evaluated Criticality Safety Benchmark Experiments, NEA/NSC/DOC(95)03/I, Nuclear Energy Agency, Paris, France (2004).

9) M. Nakagawa, T. Mori, "Whole core calculations of power reactors by use of Monte Carlo method,” J. Nucl. Sci. Technol., 30[7], 692-701 (1993).

10) J. A. Favorite, “An alternative implementation of the differential operator (Taylor series) perturbation method for Monte Carlo criticality problems," Nucl. Sci. Eng., 142, 327-341 (2002).

11) H. J. Shim, C. S. Gil, C. H. Kim, "Nuclear data sensitivity and uncertainty analysis using adjoint flux estimated in Monte Carlo forward calculations," Proc. International Conference on Nuclear Data for Science and Technology 2010, Jeju, Korea, April 26-30, 2010 (2010).

12) Y. Nagaya, T. Mori, "Estimation of reactivity worth with differential operator sampling method," Proc. Supercomputing in Nuclear Applications and Monte Carlo 2010, Tokyo, Japan, October 17-21, 2010 (2010), [USB Memory]. 